\title{
Testing surfactants as additives for clay improvement: compaction and suction effects
}

\author{
Nathan Jones ${ }^{1}$, Sérgio D.N. Lourenço ${ }^{2, a}$ and Alison Paul $\left.\right|^{3}$ \\ ${ }^{1}$ Amey, United Kingdom \\ ${ }^{2}$ Department of Civil Engineering, The University of Hong Kong, Hong Kong SAR, China \\ ${ }^{3}$ School of Chemistry, Cardiff University, United Kingdom
}

\begin{abstract}
This paper presents an exploratory study on surfactants as additives to improve soil properties. It is hypothesized that surfactant molecules populate the air-water interfaces reducing surface tension and suction thus allowing a control of the mechanical response of the soil. Suction measurements by means of a high suction tensiometer, compaction tests and Atterberg limits were conducted in mixtures of sand and kaolin, with and without a surfactant solution. The results revealed a prominent effect on suction, but to a lesser extent on the Atterberg limits and compaction behavior (the maximum dry density). This targeted effect of the surfactants suggests its molecules populate, not only the air-water interfaces decreasing surface tension, but may be adsorbing to the clay particles and forming micelles in the pore water as well. Therefore the interplay between the three may influence the soil behavior.
\end{abstract}

\section{Introduction}

\subsection{Surfactants}

Surfactants molecules consist of one polar, hydrophilic head group (water-loving) and one non-polar, hydrophobic tail group (water-hating). Surfactants have an affinity for a surface, forming into an orientated monolayer at the interface and reducing the interfacial tension. When the surface has no more space they begin to self-associate to form aggregates inside the bulk of the liquid (micelles). Micelles typically contain between 20100 surfactant molecules. The concentration of surfactants, at which micelles begin to form, is defined as the critical micelle concentration (CMC). The CMC depends on surfactant type/chemistry, temperature (Rosen, 1989) among other factors.

The chemical affinity of a surfactant with a clay depends on the basic structure of the surfactant. Surfactants are classified into three groups according to the nature of the hydrophilic portion of the molecule: (1) anionic surfactants (negatively charged head group); (2) cationic surfactants (positively charged head group); (3) nonionic surfactants (non-charged) (Rosen, 1989). Sanchez-Martin et al. (2008) found that polar surfactant adsorption towards clays would stabilize, whereas nonionic surfactant adsorption (including Triton X-100) would continue to increase above the CMC. SanchezMartin et al. (2008) also found cationic surfactants to be the most adsorbent across a variety of clay soil types. Wang et al. (1999) studied cationic surfactants and their adsorption behaviour with kaolinite and found that once the surfactant $\mathrm{CMC}$ had been reached adsorption levels would level off, suggesting that micelle formation can interrupt further adsorption. Instead, the highest adsorption levels occurred at concentrations just below the CMC. The chain length of surfactant tail groups and the chain structure within these groups may also influence the adsorption potential of surfactants. Clay surface charge distribution is sensitive to changes in chain lengths. Single chained cationic surfactants, for example, have shown a significantly greater adsorption potential towards kaolinite in comparison with double chained cationics at similar concentrations (Wang et al, 1999).

Surfactants control surface tension. Surface tension is the measure of the cohesive energy present at an interface. It is quantified as the force per unit length acting on an imaginary line drawn in the interface. For liquid water, at room temperature, the surface tension is $72 \mathrm{mNm}^{-1}$. Surface tension is important in unsaturated soils because matric suction depends, not only, on the radii of small water menisci at the interparticle contacts but also on the surface tension, where a reduction in surface tension equates to a reduction in suction. Since suction is a key variable controlling unsaturated soil behavior, being able to regulate suction via surface tension offers a series of new opportunities to use surfactants as additives for ground improvement (reduced volumetric changes, for instance).

\footnotetext{
a Corresponding author: lourenco@hku.hk
} 


\subsection{Surfactants and soil behaviour}

Among the relevant literature on the effect of surfactants on soil behaviour, it has been shown that surfactants can influence fluid density and viscosity, thus speeding up or slowing down the apparent hydraulic conductivities of soils (Lee et al, 2002). Depending on the properties and concentration of a surfactant used and clay interlayer electrostatics, Akbulut et al. (2012) revealed that surfactants can cause both flocculation and dispersion of clay particles. For example, cationic surfactants CTAC and QEFA were found to produce a dramatic change in clay plasticity. Within the effect of surfactants on soil compaction, Berney et al. (2003) assessed the influence of a wetting agent on compaction, and found that the soil with the surfactant solution achieved a higher maximum dry density at the optimum water content than the sample only with water. The Authors justified it based on a reduction of suction. However, no evidence was provided. More recently, Gluck (2010) concluded that the observed changes in the maximum dry density on various compacted granular samples with and without a commercial detergent were within possible experimental error.

In order to test the potential benefits of surfactants for ground improvement, such as an increase in maximum dry density during compaction, their effects on various soils and properties were tested in the laboratory. The remit in this study is to investigate the effect Merpol A, a non-ionic surfactant, on some soil parameters (consistency, compaction and suction) on different compositions of kaolin and sandy soils at concentrations of $0.05 \%$ and $1.0 \%$. The composition of kaolin to sand ranged from 100:0, to 50:50 and 25:75, respectively.

\section{Materials and Methods}

\subsection{Surfactant and soil}

Merpol A is described as a nonionic, polyethylene glycol phosphate ester, non-foaming wetting agent (SigmaAldrich, 2010). A surface tension of $26 \mathrm{mN} / \mathrm{m}^{-1}$ at $0.1 \%$ aqueous volume, and critical micelle concentration of $0.005 \%$ is provided in the product specification.

All tests were conducted with samples of Speswhite kaolin (100\% kaolin) and mixtures of sand and kaolin at ratios of $50 \%$ kaolin : $50 \%$ sand and $25 \%$ kaolin : $75 \%$ sand, at surfactant concentrations of $0.05 \%$ and $1 \%$, both above the critical micelle concentration. Prior to all tests, both the sand and clay were oven-dried.

\subsection{Consistency and compaction tests}

To determine the consistency behavior, the liquid limit (LL) was obtained following the Cone Penetrometer Method (BS1377:Part 2:1990:4.3) with LL corresponding to a penetration of $20 \mathrm{~mm}$ while the plastic limit (PL) also followed BS1377 with PL obtained for a water content of a thread of soil that crumbles at $3 \mathrm{~mm}$.
To determine the compaction behavior, testing followed BS1377, with dynamic compaction in three layers at increasing water contents. Selected compacted samples were kept in the compaction mould with the bottom and upper surfaces sealed with paraffin wax for suction measurement.

\subsection{Measurement of matric suction}

Suction was measured with a high suction tensiometer (Ridley \& Burland, 1993), manufactured at Cardiff University, with a $1500 \mathrm{kPa}$ air-entry value ceramic stone (Figure 1). The tensiometer used in this research measured up to $1000 \mathrm{kPa}$, its suction at cavitation.

The tensiometer was pressurized to the range 2-3 $\mathrm{MPa}$, and the measurement range tested by leaving the stone to dry to the atmosphere. The tensiometer was considered saturated, if suction at cavitation reached 1000 $\mathrm{kPa}$. If not, the instrument was placed back in the pressure vessel and repressurized. No calibration in the negative range was conducted. The calibration from the positive range was extended to the negative range.

Suction was measured at constant water content conditions by smearing the stone against the soil and waiting for equilibrium. For drier samples, the stone was covered on thin film of water to force the contact with the soil. For accuracy, measurements were conducted with two separate tensiometers. Prior to the measurements, the tensiometer was removed from the vessel and left in free water for 2 to 3 days to allow the metal body to recover from the pressure vessel confinement. Further details and justifications for the procedures are provided in Lourenço et al (2009).

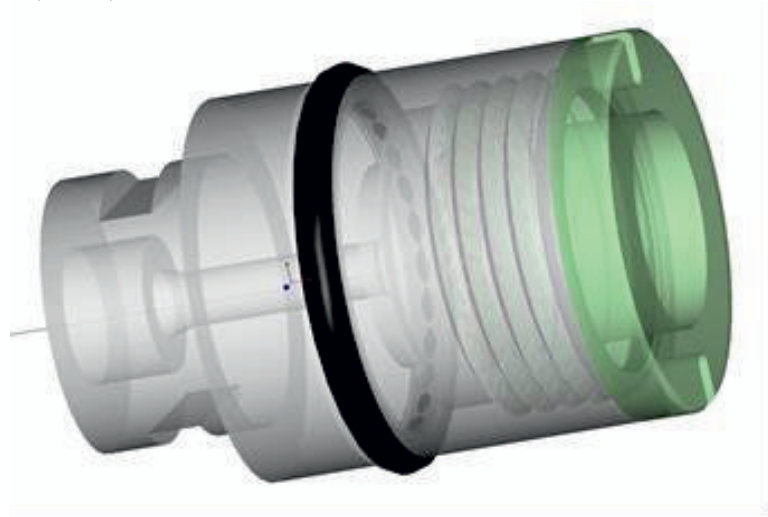

Figure 1. The high suction tensiometer used (diameter $=14 \mathrm{~mm}$; length $=22 \mathrm{~mm}$ ) (Image: Dr Xiangwei Wang, Rolls-Royce Aviation \& Aerospace).

\section{Results and Discussion}

\subsection{Consistency behaviour}

Figure $2 \mathrm{a}$ and $2 \mathrm{~b}$ show the effect of surfactant concentration on the plastic and liquid limit, respectively. The increasing concentration of surfactant, from $0 \%$ to $1 \%$, increased the plastic limit for the three soil mixtures. For the kaolin sample, the plastic limit increased from $35.1 \%$ to $42.6 \%$ while for the $25 \%$ kaolin sample, the 
increase was from $14.5 \%$ to $18.2 \%$. For the liquid limit, the results were not conclusive, with LL remaining unchanged for the kaolin sample, increasing for the 50\% kaolin sample and increasing and decreasing for the $25 \%$ kaolin sample.
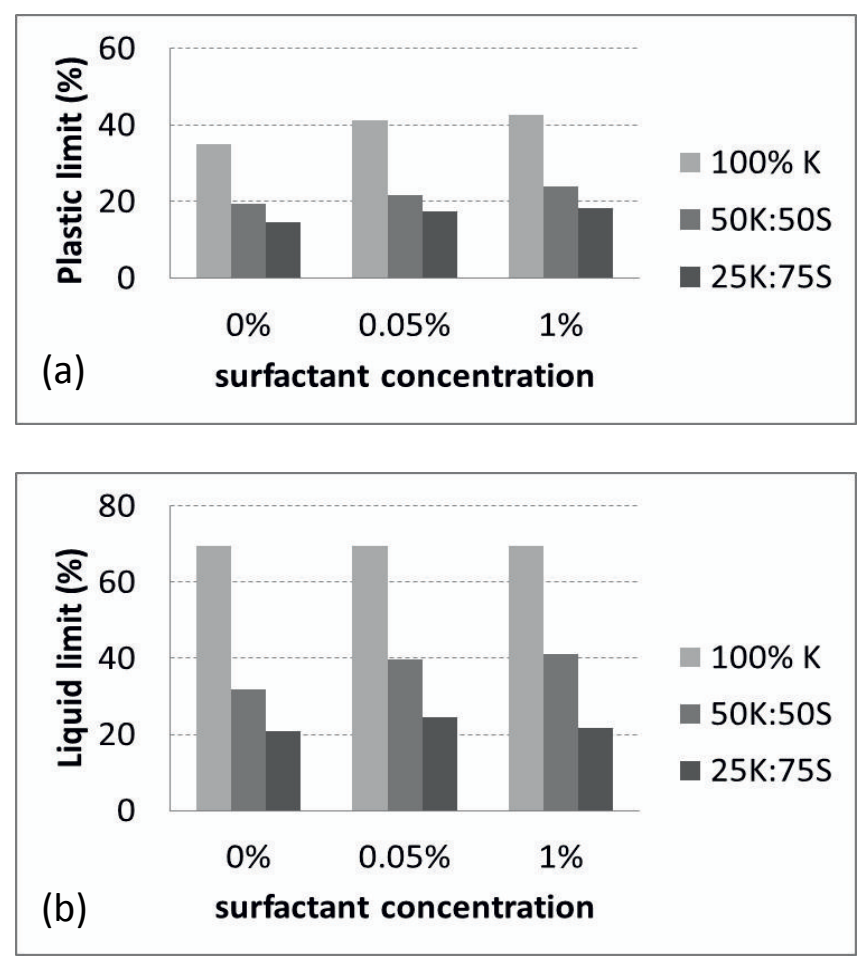

Figure 2. Effect of the surfactant concentration in the consistency behavior of three soil mixtures ( $\mathrm{K}=$ kaolin, $\mathrm{S}=$ sand). (a) Plastic limit; (b) Liquid limit.

\subsection{Compaction behaviour}

Figures $3 \mathrm{a}, 3 \mathrm{~b}$ and $3 \mathrm{c}$ show the compaction curves at different surfactant concentrations $(0 \%, 0.05 \%, 1 \%)$ for $10 \%$ kaolin, $50 \%$ kaolin and $25 \%$ kaolin, respectively. Figure 3a, for kaolin, shows tests at two further concentrations at $0.1 \%$ and $4.5 \%$. The compaction curves revealed no particular trend, with the curves overlapping for the $50 \%$ and $25 \%$ kaolin samples. Differences in the maximum dry density and optimum water content between the three figures are due to the material, rather than surfactant concentration. The only exception is for the kaolin samples, where the maximum dry density dropped by $0.05 \mathrm{Mg} / \mathrm{m}^{3}$ for the samples prepared at higher surfactant concentrations (1\% and 4.5\%). This finding contradicts the results by Berney et al. (2003) where the maximum dry density was increased with the surfactants.
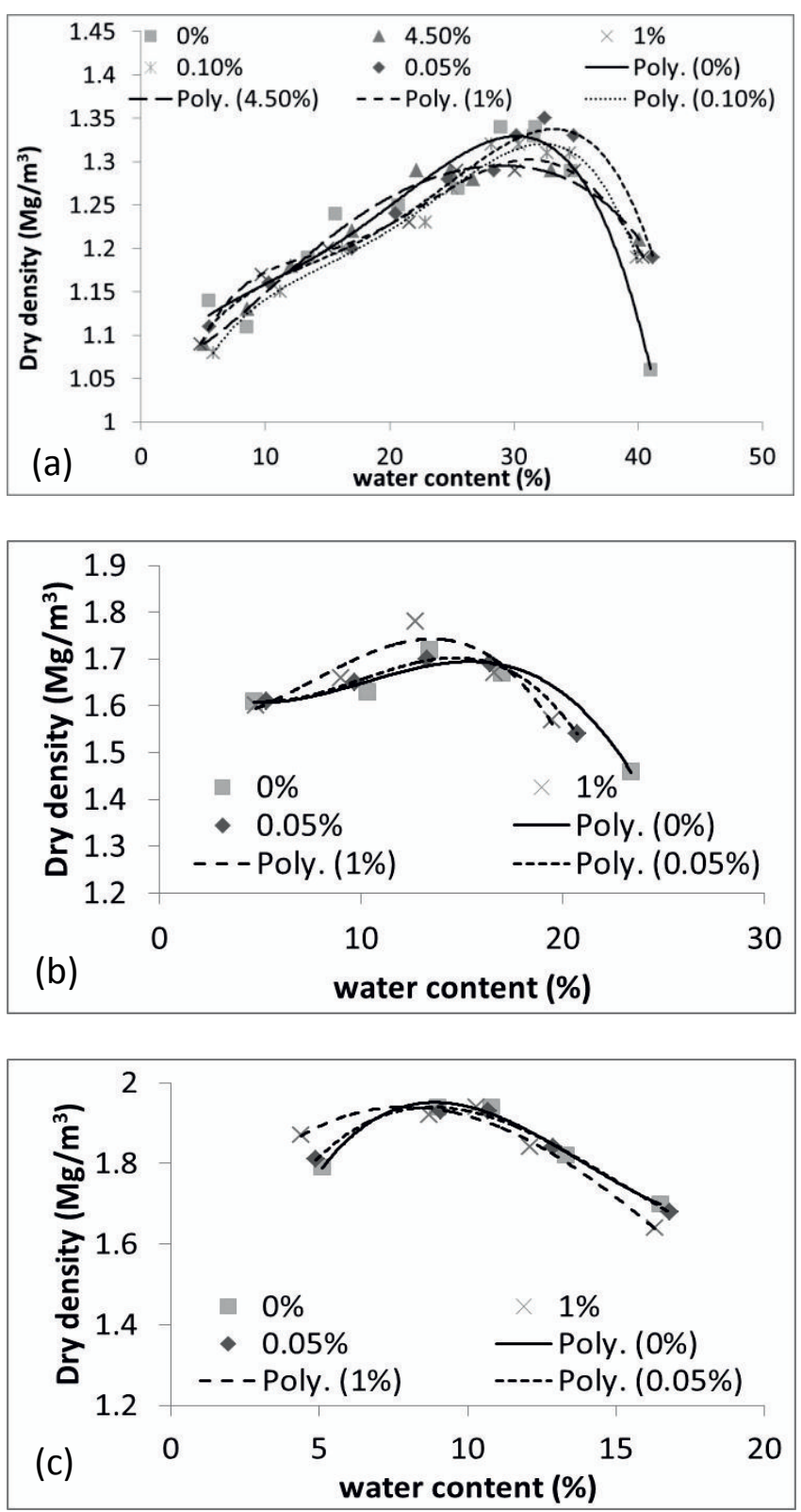

Figure 3. Compaction curves at different surfactant concentrations for (a) 100\% kaolin, (b) 50\% kaolin : 50\% sand, (c) $25 \%$ kaolin : $75 \%$ sand.

\subsection{Suction behaviour}

Figure 4a shows suction against the surfactant concentration for a $50: 50$ sample at $10 \%$ and $15 \%$ water content. At $10 \%$ water content, there was a reduction of $235 \mathrm{kPa}$ by adding $0.05 \%$ surfactant and $800 \mathrm{kPa}$ when adding $1 \%$ surfactant solution. At $15 \%$ water content, the reduction in suction was smaller because of the higher water content. Figure $4 \mathrm{~b}$ shows suction against the surfactant concentration for a 75:25 sample at 5\% and $10 \%$ water content. The results are similar to Figure 2, except that given the higher sand content, the water content was smaller (5\%) to achieve high suctions $(1000 \mathrm{kPa}$ for the sample without surfactant). These results confirm that the surfactants reduce suction, likely linked to a reduction in the surface tension. 

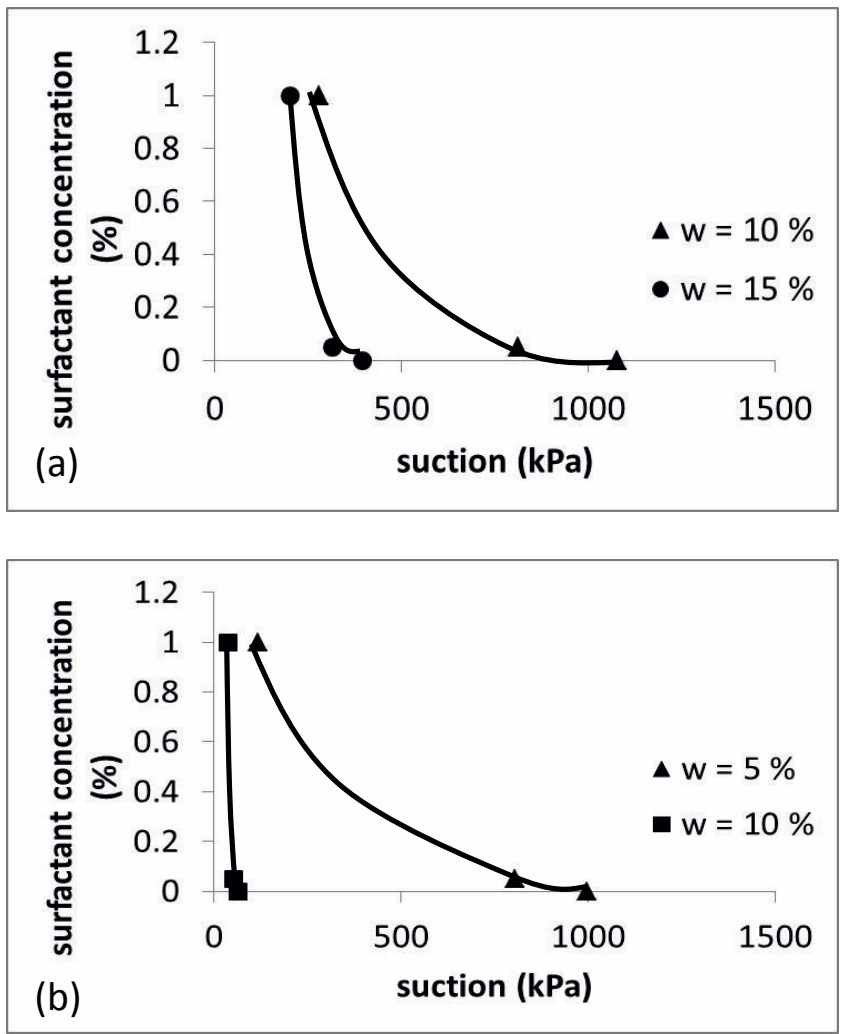

Figure 4. Effect of surfactant concentration on suction for (a) 50\% kaolin : 50\% sand, and (b) 25\% kaolin : $75 \%$ sand.

\subsection{Summary}

This preliminary study revealed no common trends between the three sets of results (consistency, compaction and suction), suggesting that the surfactants have specific effects in certain soil properties. The presence of surfactants had an effect in the plastic limits and suction behaviour, but was inconclusive in the compaction behaviour and liquid limit. Increasing surfactant concentrations decreased suction and increased the plastic limits. A reduction in the maximum dry density did occur but only at high surfactant concentrations.

The compaction results revealed that the explanation proposed by Berney et al. (2003) whereby the maximum dry density is increased due to a reduction in suction does not hold for all soils and conditions. The maximum dry density changed little even when suction was reduced by $80 \%$ at $1 \%$ surfactant concentration.

This study also revealed that the CMC, an important property of surfactants, does not act as a threshold in the soil behavior. If it did, soil properties would not change for concentrations higher than the CMC. But, as shown for all datasets, increasing concentrations past the CMC had an effect on suction, plastic limit and compaction. This suggests that surfactant molecules populate, not only the air-water interfaces decreasing surface tension, but may be adsorbing to the clay particles and forming micelles in the liquid phase. Therefore the interplay between the three, may affect soil behavior.

\section{Conclusions}

The paper presents an experimental study on the behavior of unsaturated soils with surfactant solutions. Suction measurements by means of high suction tensiometers, compaction tests and the Atterberg limits were conducted in mixtures of sand and kaolin, with and without surfactant solutions. Compaction showed a decrease in the maximum dry density for the $100 \%$ kaolin sample at higher surfactant concentrations. The change in the maximum dry density data between the different concentrations was negligible or inconclusive. Plastic limits grew significantly with increasing surfactant concentration. Suction consistently reduced with increasing surfactant concentrations, even past the CMC, which suggests that surface tension cannot be the only mechanism responsible for decreasing suction. Other factors, such as the adsorption of surfactant molecules to the clay particles and micelle formation in the pore water may also affect soil behaviour.

\section{Acknowledgements}

Funding to develop the high-suction tensiometer was provided via a UK Royal Society Research Grant RG090044. Manufacturing was conducted by Dr. Xiangwei Wang (Manufacturing Engineering Centre, Cardiff University, UK, now Rolls-Royce Aviation \& Aerospace, Bristol, UK).

\section{References}

1. Z. Akbulut, N. Kurt, S. Arasan. Earth Sciences Resource Journal 16 (2):13-19 (2012)

2. E. Berney, J. Peters, K. Newman, D. Smith. Transportation Research Record: Journal of the Transportation Research Board, 1819:57-62 (2003)

3. O. Gluck. MSc. Dissertation, Cardiff University, pp. 117 (2010)

4. D.-H. Lee, R.D. Cody, D.-J. Kim, S. Choi. Environment International 27, 681-688 (2002)

5. S.D.N. Lourenço, D. Gallipoli, D. Toll, F. Evans. ASTM Geotechnical Testing Journal 32, 6, 1-7 (2009)

6. A. Ridley, J. Burland. Géotechnique 43 (2), 321-324 (1993)

7. M.J. Rosen. Surfactants and interfacial phenomena, 2nd edition, John Wiley \& Sons Inc. (1989)

8. M.J. Sánchez-Martín, M.C. Dorado, C. del Hoyo, M.S. Rodríguez-Cruz. Journal of Hazardous Materials 150, 115-123 (2008)

9. J. Wang, B. Han, M. Dai, H. Yan, Z. Li, R.K. Thomas. Journal of Colloid and Interface Science 213, 596-601 (1999) 